\title{
GANGLIONEUROBLASTOMA OF THE THORACIC SPINAL CORD: A VERY RARE CASE REPORT
}

\author{
Bekir Akgun ${ }^{1}$,Deniz Ates ${ }^{2}$,Metin Kaplan ${ }^{1}$
}

Firat University Hospital, Department of Neurosurgery, Elazig, Turkey ${ }^{1}$; Hacettepe University Hospital, Department of Pathology, Ankara, Turkey²

\begin{abstract}
Summary: An eight-month-old male child was admitted with weakness and swelling in the feet. Paraparesis and bilateral lower extremity edema were present in the neurological examination. Thoracic MRI showed an intradural intramedullary mass $61 \times$ $11 \mathrm{~mm}$ in size in the T5-T10 levels. Laminotomy between the T5-T10 vertebrae was performed. A mass with smooth borders was separated from most of the neural tissue. In the postoperative MRI, we observed a contrast enhancing area, considered a residual fragment, only $5 \times 4 \mathrm{~mm}$ in size. Histopathological properties were compatible with the intermixed subtype of ganglioneuroblastoma. Only a limited number of thoracic cord Ganglioneuroblastoma reports have been previously published. Although very rare in children and young adults, ganglioneuroblastoma should be included in the differential diagnosis of thoracic cord tumors. It is difficult to obtain a preoperative diagnosis with clinical features and radiological investigations. Diagnosis depends on histopathological examinations. Curative treatment should be in the form of a complete resection of the tumor. In partially resected cases, adjuvant radiotherapy may become necessary, along with close follow-up.
\end{abstract}

Key words: Ganglioneuroblastoma; Intramedullary tumor; Thoracic cord

\section{Introduction}

Neuroblastic tumors derive from primordial neural crest cells which constitute the sympathetic nervous system (1). Peripheral neuroblastic tumors are categorized as Ganglioneuromas (GNs), Ganglioneuroblastomas (GNBs), and Neuroblastomas (NBs) (3). GNs are the most mature forms. Ganglioneuroblastomas are tumors containing both primitive neuroblasts and mature ganglion cells are potentially malignant, although less aggressive, than neuroblastomas (2).

Central nervous system GNBs are uncommon. Thoracic spinal cord localization is very rare (5). In this report, we present the case of a child with thoracic spinal cord ganglioneuroblastoma and discuss the condition's clinical behaviour, radiological properties, histopathological features, and treatment modalities.

\section{Case report}

An eight-month-old male child was admitted with weakness and swelling in the feet which had been present for one month previously. In the neurological examination, paraparesis and bilateral lower extremity edema were present. Thoracic MRI revealed an intradural intramedullary spinal cord mass, $61 \times 11 \mathrm{~mm}$ in size, in the T5-T10 levels (Figure 1). No other lesion was detected after the screening of the entire spine and the brain. The patient underwent surgery.
Laminotomy between T5-T10 vertebrae was performed. A mass with smooth borders was separated from most of the neural tissue. We had thought that the tumor had been totally resected and that we had not left residual mass intraoperatively; however, a $5 \times 4 \mathrm{~mm}$ sized contrast enhancing area in the T5-6 levels was observed in postoperative MRI. This was considered a residual fragment (Figure 2).

Hematoxylin and eosin stained sections showed a partially necrotic neoplasm encircling the peripheral nerves. The neoplasm was composed of microscopic nests of neuroblastic cells intermingled in the ganglioneuromatous stroma. These nests were composed of a mixture of neuronal cells in various stages of differentiation, i.e. differentiating neuroblast from maturing ganglion cells within a neuropil background. Two large neuroblastic nodular components showing hypercellularity and increased mitotic activity were present (Figure 3).

Immunohistochemically neuronal cells showed variable expression by neuronal markers i.e. NFP, synaptophysin, and GFAP. The Ki-67 labeling index was high in the hypercellular neuroblastic nodules. These histopathological properties were compatible with the intermixed subtype of ganglioneuroblastoma. In the early post-operative period, some improvements were observed in the patient's paraparesis and lower extremity edema. The patient, whose follow-up has been ongoing over the last six months, has not experienced any postoperative neurological deterioration so far. 


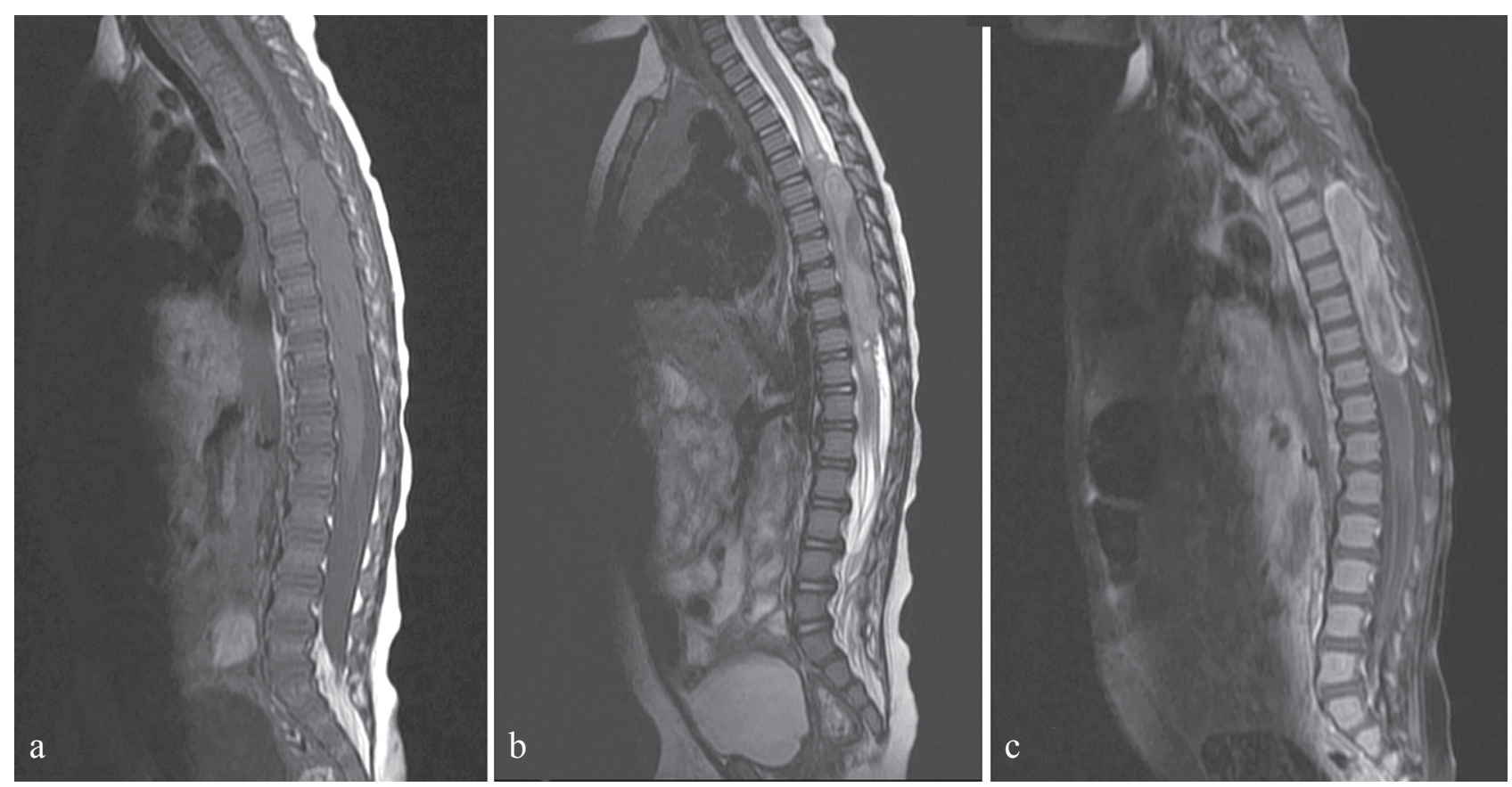

Fig. 1: The patient's thoracic MRI represented intradural intramedullary mass, in T5-T10 levels. (a) Lesion was hypointense on T1-weighted images, (b) heterogeneous hyperintense on T2-weighted images, (c) enhanced with contrast material strongly. Spinal cord was compressed from posterior to anterior by the mass as it had grown posteriorly.
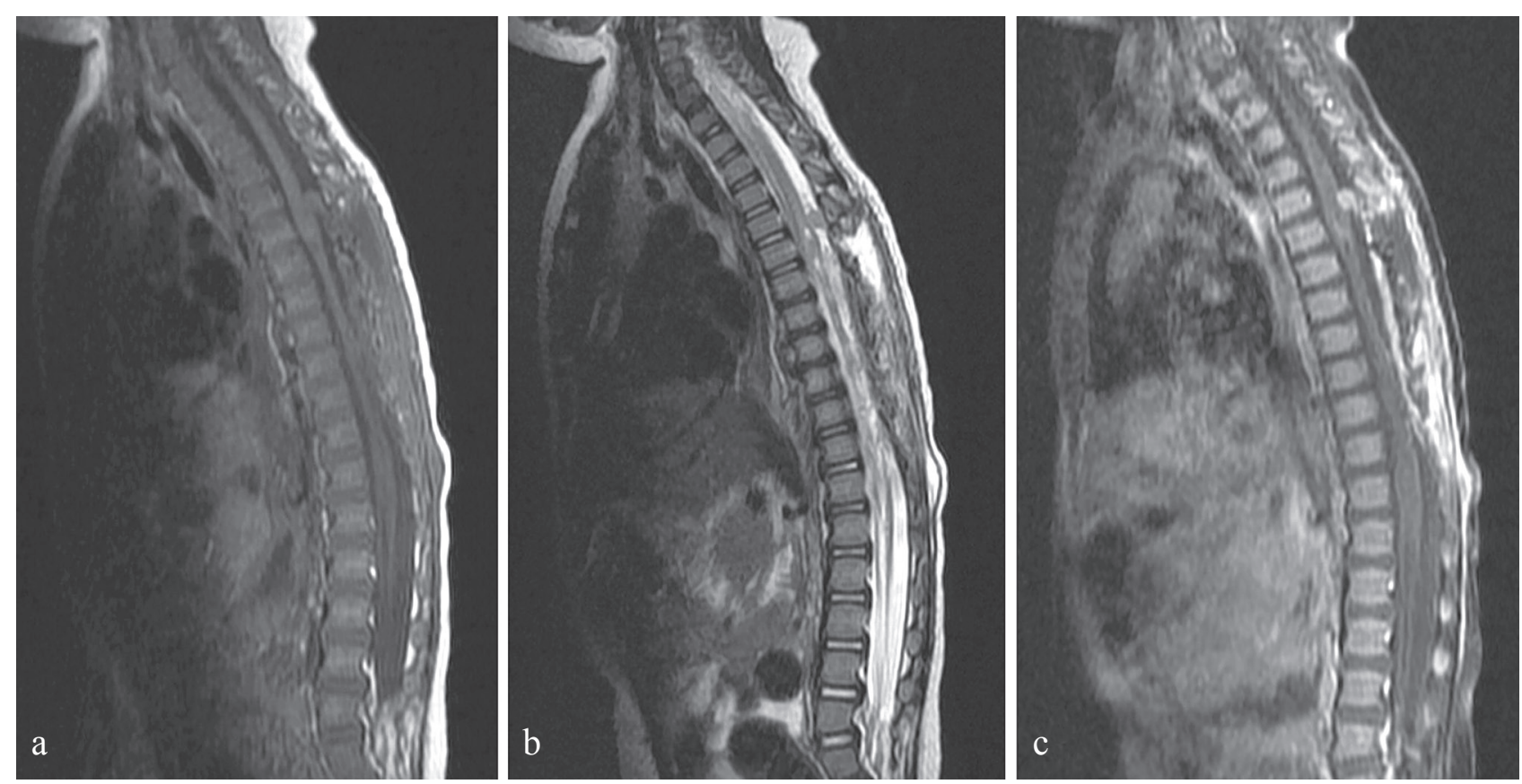

Fig. 2: Postoperative MRI showed the tumor had been resected but in T5-6 levels, a $5 \times 4$ mm sized hypointense area was observed on (a) T1-weighted images and (b) T2-weighted images. (c) This area was enhanced with contrast material, and thus was thought to be a residual fragment. 


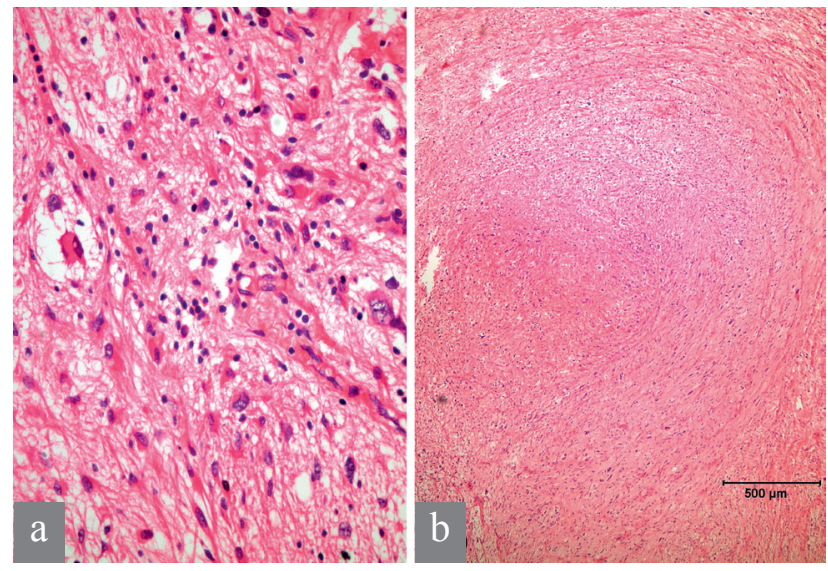

Fig. 3: (a) Intermixed subtype of ganglioneuroblastoma area which was rich with schwannian stroma. (b) Large neuroblastic nodular component.

\section{Discussion}

Ganglioneuroblastomas are rare childhood neoplasms. They can also appear in adults, but more rarely. More than $90 \%$ of cases are reported in children younger than five years of age. There are two subtypes described in existing literature: the nodular subtype includes gross nodules of neuroblastoma (immature small cells) in wide expanses of ganglioneuroma (large mature cells in a fusiform stroma), whereas the intermixed subtype consists of microscopic nests of neuroblastoma settled in a ganglioneuromatous stroma (4).

GNBs occur wherever sympathetic nervous systems are present. Mediastinum and retroperitoneal tissues are the most common sites for ganglioneuroblastomas $(1,2)$.

Central nervous system GNBs are uncommon. They can arise anywhere in the central nervous system. The cerebral hemispheres, the floor of the third ventricle, pineal gland, and cerebellum are some instances of the localizations of central nervous system GNBs $(1,5)$. In our case, the tumor was located in the thoracic spinal cord. Localization in the spinal cord, particularly in the thoracic spinal cord, is very rare. Only a limited number of thoracic cord Ganglioneuroblastoma reports have been previously described. In light of the existing literature, the most common spinal localization for GNBs is the cervical cord, which represents the route of their spreading from the medulla. For this reason, in these kinds of cases, it is necessary to investigate the brain. Notably, imaging of the posterior fossa is very important (5).

Gait disturbance and paraparesis are the most common symptoms in thoracic cord neuroblastic tumors such as other tho- racic cord neoplasms. Also, leg length discrepancy and scoliosis were reported (5). Our patient had paraparesis and leg edema.

GNB is nonaggressive. According to the reported cases, tumor growth is slow. The prognosis of this neoplasm is usually favorable. Surgical excision is often the most effective treatment. This treatment can be followed by radiotherapy only when continued tumor growth has occurred (5).

The most common intramedullary neoplasms are astrocytomas and ependymomas. Preoperative differentiation of tumors is usually difficult; both histologic types can be solid, extensive or cystic with nodules (5). In the present case, the MRI findings showed an intramedullary, solid tumor that was hypointense on T1-weighted images and heterogeneous hyperintense on T2-weighted images. It was intensively enhanced with contrast material. The clinical behavior was also nonspecific. These features make it difficult to differentiate this lesion from other kinds of intramedullary tumors. Neuroblastic tumors should be considered in the differential diagnosis of intramedullary tumors, even if they are uncommon.

\section{Conclusion}

In conclusion, even though very rare, ganglioneuroblastoma in children and young adults should be included in the differential diagnosis of thoracic cord tumors. It is not easy to realize a specific preoperative diagnosis with a clinical picture and radiological studies; therefore, definitive diagnosis depends on postoperatively performed histological examination and pathological diagnosis. Curative treatment should be a complete resection of the tumor. Nonetheless, in partially resected cases, adjuvant radiotherapy may become necessary, along with close follow-up. Also, radiotherapy may be proposed if it is appropriate for the patients and suitable for their age and performance.

\section{References}

1. Gasparetto EL, Rosemberg S, Matushita H, Leite CdC. Ganglioneuroblastoma of the cerebellum: neuroimaging and pathological features of a case. Arq. Neuro-Psiquiatr 2007; 65(2A): 338-40.

2. Mizuno S, Iida T, Fujita S. Adult - Onset Adrenal Ganglioneuroblastoma - Bone Metastasis Two Years After Surgery: Report of a Case. Surg Today 2010; 40: 482-6.

3. Okamatsu C, London WB, Naranjo A, et al. Clinicopathological Characteristics of Ganglioneuroma and Ganglioneuroblastoma: A Report from the CCG and COG. Pediatr Blood Cancer 2009; 53: 563-9.

4. Peycru T, Guiramand J, Tardat E, Savoie PH, Avaro JP, Balandraud P. Nodular ganglioneuroblastoma in adults. Can J Surg 2009; 52(4): 111-3.

5. Sibilla L, Martelli A, Farina L, et al. Ganglioneuroblastoma of the Spinal Cord. AJNR 1995; 16: 875-7.

Received: 11/06/2011

Accepted in revised form: 18/12/2011

\section{Corresponding author:}

Dr. Bekir AKGUN, Firat Universitesi, Tip Fakultesi, Firat Tip Merkezi, Beyin Cerrahi Klinigi, 23200, Elazig, Turkey; e-mail: bekirakgun@yahoo.com 